ORIGINAL ARTICLE

\title{
Estudo da transferência de massa de cortes de frango durante o tratamento osmótico em diferentes soluções salinas
}

\section{Study of mass transfer during osmotic treatment of chicken cuts with different salt solutions}

\author{
Natália Stevanato ${ }^{1}$, Barbara Daniele Almeida Porciuncula ${ }^{1 *}$ (i) \\ ${ }^{1}$ Universidade Estadual de Maringá (UEM), Departamento de Tecnologia, Umuarama/PR - Brasil
}

*Corresponding Author: Barbara Daniele Almeida Porciuncula, Universidade Estadual de Maringá (UEM), Departamento de Tecnologia, Av. Angelo Moreira da Fonseca, 1800, CEP: 87506-370, Umuarama/PR -

Brasil, e-mail: barbara.porciuncula@gmail.com

Cite as: Stevanato, N., \& Porciuncula, B. D. A. (2020). Study of mass transfer during osmotic treatment of chicken cuts with different salt solutions. Brazilian Journal of Food Technology, 23, e2019295. https://doi.org/10.1590/1981 6723.29519

\begin{abstract}
Resumo
A adição de cloreto de potássio $(\mathrm{KCl})$ tem sido proposta como substituto de cloreto de sódio $(\mathrm{NaCl})$ em produtos cárneos. O objetivo do presente trabalho é estudar a transferência de massa (TM) de cortes de peito frango imersos em soluções salinas com diferentes formulações de $\mathrm{NaCl}$ e $\mathrm{KCl}$, durante o tratamento osmótico. Os experimentos foram conduzidos em diferentes soluções salinas utilizando três formulações: (A) $100 \% \mathrm{NaCl}$, (B) $50 \%$ de $\mathrm{NaCl}$ e $50 \%$ de $\mathrm{KCl}$, e (C) $100 \% \mathrm{KCl}$, sob agitação, numa proporção de 1:10 (m/m) de sal:água e temperatura da solução de aproximadamente $1{ }^{\circ} \mathrm{C}$. A TM foi caracterizada por meio da determinação dos parâmetros de ganho de água (GA), ganho de sal (GS), ganho de massa (GM) e coeficiente de difusão aparente da água $\left(D_{a p}\right)$, em cortes de frango. Os resultados mostraram que a substituição do $\mathrm{NaCl}$ por $\mathrm{KCl}$ foi efetiva, visto que, com a formulação (B), a carne de frango apresentou maior absorção de sal quando comparada com a formulação (A). No final de $6 \mathrm{~h}$ de salga, as amostras apresentaram incremento entre 10,5\% e 11,0\% de GA, não apresentando diferenças significativas entre os tratamentos. A formulação (B) proporcionou maior GS pelas amostras, atingindo $3,77 \mathrm{~g}$ sal $100 \mathrm{~g}^{-1}$ de amostra inicial e, consequentemente, maior coeficiente de difusão aparente da água na carne. A formulação (A) apresentou-se inferior quanto aos parâmetros de GS e GM, quando comparada com as demais formulações, devido ao efeito osmótico menos intenso provocado por ela.
\end{abstract}

Palavras-chave: Tratamento osmótico; Soluções salinas; Cloreto de sódio; Cloreto de potássio.

\begin{abstract}
Potassium chloride $(\mathrm{KCl})$ can be used as a substitute for $\mathrm{NaCl}$ in meat products. The aim of this work was to study the mass transfer (MT) during osmotic treatment of chicken breast cuts immersed in salt solutions of varying amounts of $\mathrm{NaCl}$ and $\mathrm{KCl}$. The experiments were carried out using salt solutions of three different formulations: (A) $100 \% \mathrm{NaCl},(B) 50 \% \mathrm{NaCl}$ and $50 \% \mathrm{KCl}$, and (C) $100 \% \mathrm{KCl}$; solutions were kept under stirring, containing 1:10 (m/m) of salt; solution temperature was of about $1{ }^{\circ} \mathrm{C}$. MT was characterized by evaluating water gain (WG), salt gain (SG),
\end{abstract}


weight gain (WG) and apparent diffusion coefficient of water $\left(D_{a p}\right)$ of chicken parts. The results showed that substitution of $\mathrm{NaCl}$ for $\mathrm{KCl}$ was effective, since with formulation (B) chicken meat showed higher salt absorption in comparison to the formulation (A). At the end of 6 hours of curing the samples showed an WG increase between $10.5 \%$ and $11.0 \%$, with no significant differences between the treatments. Samples treated with formulation (B) had higher SG values, totaling $3.77 \mathrm{~g}$ salt $100 \mathrm{~g}^{-1}$ of initial sample and consequently led to a higher apparent diffusion coefficient of water in the meat. Treatment with formulation (A) led to lower WG and GS parameters when compared with the other formulations due to less intense osmotic effect caused by this.

Keywords: Osmotic treatment; Salt solutions; Sodium chloride; Potassium chloride.

\section{Introdução}

A avicultura brasileira é considerada dinâmica e vem se colocando entre as mais desenvolvidas do mundo (Ziebert \& Skikida, 2004). Segundo os dados da Associação Brasileira de Proteína Animal (2018), em 2017, o consumo per capita de carne de frango chegou a $42,07 \mathrm{~kg} /$ habitante. O Brasil é o maior exportador de aves do mundo, exportando 4,320 mil toneladas de carne de frango, e é o segundo produtor mundial, com 13,056 mil toneladas produzidas, em 2017.

A salga é um dos principais métodos empregados na conservação de carnes e derivados. Uma importante função conservadora do cloreto de sódio na indústria de produtos cárneos é diminuir a atividade de água do material e, portanto, reduzir o crescimento microbiano (Aliño et al., 2010). Além disso, o sal promove a extração das proteínas miofibrilares, como a actina e a miosina. A extração e a solubilização dessas proteínas musculares contribuem para a emulsificação das gorduras e o aumento da sua capacidade de retenção de água, o que reduz perdas de peso durante o cozimento. Como consequência disso, há um aumento na maciez e suculência da carne, contribuindo assim para melhorar a qualidade e a textura do produto (Zheng et al., 2019; Desmond, 2006; Silva Sobrinho et al., 2004).

A taxa de remoção de água em alimentos depende de variáveis e parâmetros do processo, tais como a temperatura, a concentração da solução osmótica, o tamanho e a geometria do material, e o nível de agitação (Rastogi \& Raghavarao, 2004). No geral, quanto maiores a concentração do soluto, o tempo de contato, a agitação e a temperatura, maior será a transferência de massa (Sabadini et al., 1998).

Durante o processo de tratamento osmótico, ocorrem, simultaneamente, dois fluxos de transferência de massa em contracorrente, sendo um a difusão da água do interior da carne para a solução hipertônica e o outro a difusão do soluto para o interior da carne. A força motriz para a difusão da água do interior do alimento para a solução osmótica é regida pela diferença na pressão osmótica (Ahmed et al., 2016; Rastogi \& Raghavarao, 2004; Sabadini et al., 1998, 2001).

A adição de sal em alimentos, em concentrações baixas, causa o aumento da capacidade de retenção de água. Porém, à medida que o sal se difunde no interior do músculo, os íons cloreto $\left(\mathrm{Cl}^{-}\right)$formam um complexo com as proteínas miofibrilares, que, por sua vez, tem a pressão osmótica maior que a solução salina, provocando um fluxo de água no sentido inverso (Pardi et al., 2004). A ação do cloreto de sódio também está relacionada com a inibição da multiplicação de microrganismos e a atividade enzimática, devido ao aumento da pressão osmótica do meio do alimento, com consequente redução da atividade de água (CostaCorredor et al., 2010; Lilic et al., 2008; Silva Sobrinho et al., 2004).

Atualmente, produtos considerados saudáveis estão recebendo cada vez mais atenção dos consumidores, que buscam produtos saborosos e convenientes; entretanto, esses consumidores também estão preocupados com o valor nutritivo, a segurança e os benefícios que o alimento possa trazer. O sal é de extrema importância nas indústrias de processamento e no preparo de produtos cárneos, e, apesar disso, diversos estudos têm mostrado que o consumo excessivo de sódio está associado com efeitos negativos à saúde, principalmente problemas de hipertensão arterial. Assim, torna-se crescente a preocupação com a redução deste ingrediente nos alimentos processados (Costa-Corredor et al., 2010; Kloss et al., 2015; Paulino et al., 2006). 
Grande parcela do sódio presente na dieta provém de alimentos industrializados e, dentre estes, se destacam os produtos cárneos e derivados, contribuindo com aproximadamente $20 \%$ da ingestão diária (Lazic et al., 2015; Lilic et al., 2008). A maior concentração deste sódio é proveniente de produtos cárneos processados, uma vez que

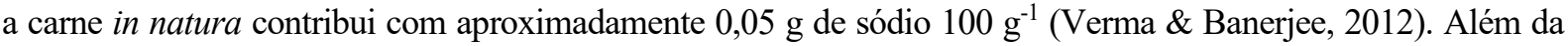
palatabilidade oriunda do gosto salgado, o cloreto de sódio é responsável por desenvolver propriedades de textura desejáveis de carnes processadas. Com isso, a indústria cárnea enfrenta um grande desafio no desenvolvimento de produtos que satisfaçam sensorialmente a expectativa dos consumidores e, ao mesmo tempo, com baixo risco nocivo à saúde relativo à ingestão de sal de sódio (Nascimento et al., 2007).

As alternativas para a diminuição do sódio nas carnes processadas envolvem a redução direta do teor de sal adicionado ou a substituição parcial do $\mathrm{NaCl}$ por sal não sódicos (Bampi et al., 2016; Ruusunen \& Puolanne, 2005; Rocha Garcia et al., 2013). Um substituto ideal do $\mathrm{NaCl}$ deve se comportar da mesma maneira na matriz da carne e deve ter efeito semelhante sobre as características do produto (Costa-Corredor et al., 2010).

$\mathrm{O} \mathrm{KCl}$ tem sido proposto como substituo parcial do $\mathrm{NaCl}$ para a redução do teor de sódio em alimentos, uma vez que este sal possui propriedades similares e é reconhecido como seguro (Ruusunen \& Puolanne, 2005). Estudos mostraram que a substituição parcial do $\mathrm{NaCl}$ por $\mathrm{KCl}$ foi realizada sem perda da funcionalidade e aceitabilidade de salsinhas de porco (37,05\%) (Stanley et al., 2017), salsichas de Harbin (30\%) (Chen et al., 2019) e jerked beef (50\%) (Vidal et al., 2019). Dessa forma, com a aplicação de $\mathrm{KCl}$, cortes de peito de frango salgado podem ser introduzidos no mercado alimentício como ingrediente proteico com baixo teor de sódio.

Com isso, o presente estudo teve como objetivo estudar a transferência de massa de solutos (ganho de água, sal e massa) em cortes de peito de frango imersos em soluções salinas com diferentes agentes osmóticos: $\mathrm{NaCl}, \mathrm{KCl}$ e uma mistura de $50 \% \mathrm{NaCl}$ e $50 \% \mathrm{KCl}$.

\section{Material e métodos}

Todos os experimentos foram realizados utilizando cortes de carne de frango congelados, sem osso e sem pele, provenientes da mesma unidade produtora e adquiridos no comércio local da cidade de Loanda-PR. O teor de água das amostras foi determinado em estufa (LS Logen ${ }^{\circledR} 1.3$ ) a $105^{\circ} \mathrm{C}$ até peso constante e o teor de proteína foi determinado pelo método de determinação de nitrogênio total, Kjeldahl, utilizando fator de conversão (f) de 6,25. (Instituto Adolfo Lutz, 2008).

\subsection{Preparo das amostras}

As amostras foram cortadas em formato retangular de 2,0 $\times 2,0 \times 1,5 \mathrm{~cm}$, como apresentado na Figura 1, e mantidas à temperatura do experimento $\left(1{ }^{\circ} \mathrm{C}\right)$ até sua utilização.

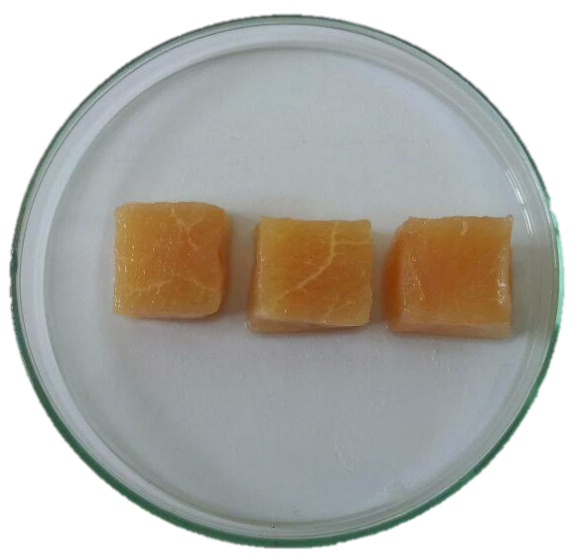

Figura 1. Amostras de corte de peito de frango utilizadas nos experimentos. 


\subsection{Tratamento osmótico}

\subsubsection{Preparo das soluções}

Foram preparadas soluções salinas utilizando três formulações: (A) $100 \% \mathrm{NaCl}$, (B) $50 \%$ de $\mathrm{NaCl}$ e $50 \%$ de $\mathrm{KCl}$, e (C) 100\% KCl, a uma razão mássica de 1:10 (m/m) de sal:água destilada (Schmidt et al., 2008a), e foram mantidas a $1{ }^{\circ} \mathrm{C}$. Utilizou-se uma proporção de 1:10 (m/v) de produto: salmoura (Sabadini et al., 2001), proporção grande o suficiente para evitar gradientes de concentração durante o processo.

\subsubsection{Procedimento experimental}

As amostras, devidamente pesadas e identificadas, foram colocadas em contato com a solução salina em um recipiente de polipropileno e acondicionadas em um refrigerador (Consul ${ }^{\circledR}$ ) com o termostato ajustado em um nível cuja temperatura corresponde a $1^{\circ} \mathrm{C}$, sendo a mesma monitorada por um termômetro digital.

Com o intuito de garantir a transferência de massa tridimensional, a solução foi agitada através de uma bomba de circulação de líquido submersa. Em tempos pré-determinados, a cada 10 min na primeira hora de experimento, a cada 30 min nas três horas subsequentes e nos tempos de 4, 5, 6 e 24 horas de processo, a amostra foi retirada da solução. Estas condições foram definidas com base no estudo de Bampi et al. (2016). Em seguida, a amostra foi colocada sobre papel absorvente para a remoção da salmoura aderida na superfície e então foi determinado o teor de água em estufa (LS Logen 1.3 ) a $105^{\circ} \mathrm{C}$ até peso constante. Os experimentos e o processo foram conduzidos em triplicada.

Os parâmetros ganho de água (GA), ganho de sal (GS) e ganho de massa (GM) foram determinados a partir das Equações 1, 2 e 3, respectivamente.

$$
\begin{aligned}
& G A=\frac{W_{w 0}-W_{w}}{W_{0}} .100 \\
& G S=\frac{W_{s}-W_{s 0}}{W_{0}} .100 \\
& G M=\frac{W_{0}-W}{W_{0}} .100
\end{aligned}
$$

em que $\mathrm{W}_{\mathrm{w} 0}$ é a massa inicial de água na amostra, $\mathrm{W}_{\mathrm{w}}$ é a massa de água na amostra ao fim do tratamento, $\mathrm{W}_{0}$ é a massa inicial da amostra, $\mathrm{W}_{\mathrm{s}}$ é a massa de sólidos secos ao fim do tratamento, $\mathrm{W}_{\mathrm{s} 0}$ é a massa inicial de sólidos secos da amostra e $\mathrm{W}$ é a massa da amostra ao fim do tratamento.

O coeficiente de difusão aparente da água $\left(D_{a p}\right)$ foi determinado a partir da solução analítica da lei de Fick desenvolvida por Crank (1975) para a placa plana, considerando a transferência de massa tridimensional e resistência externa desprezível, pela Equação 4:

$\frac{W_{0}^{i}-W_{t}^{i}}{W_{0}^{i}-W_{e q}^{i}}=\sum_{n=0}^{n=8} \frac{8}{(2 n+1)^{2} \pi^{2}} e\left[\frac{-F_{0}(2 n+1)^{2} \pi^{2}}{4}\right]$

Sendo

$F_{0}=\frac{D_{a p} t}{L^{2}}$

em que $W$ representa a fração mássica da água na amostra e os subíndices representam o tempo inicial $(0)$, o tempo decorrido $(t)$ e o tempo infinito $(e q) ; F_{o}$ é o número de Fourier (adimensional); $n$ é o número de termos da série, $D_{a p}$ é a difusividade aparente $\mathrm{em}^{2} \mathrm{~s}^{-1}, L$ é a espessura da amostra em $\mathrm{m}$. Note-se que a $W_{e q}$ das amostras de corte de frango foi determinada após $24 \mathrm{~h}$ de imersão nas salmouras. 


\subsection{Análise estatística de dados}

A avaliação das diferenças entre os valores de ganho de água (GA), ganho de sal (GS) e ganho de massa (GM) das amostras, nos diferentes tratamentos, foi realizada a partir da Análise de Variância (ANOVA) com nível de significância de 95\% $(\alpha=0,05)$, com auxílio do software STATISTICA 8.0 (STATSOFT ${ }^{T M}$, Inc.).

\section{Resultados e discussões}

A carne de frango in natura utilizada neste trabalho foi caracterizada quanto ao teor de proteína e água, como apresentado na Tabela 1.

Tabela 1. Propriedades dos cortes de peito de frango in natura.

\begin{tabular}{cl}
\hline Teor de água ( $\left(\mathrm{g}\right.$ de água $\mathrm{g}^{-1}$ sólidos secos) & Teor de Proteína (\%) \\
\hline $2,823 \pm 0,002$ & $20,51 \pm 0,28$ \\
\hline
\end{tabular}

Valores semelhantes de teor de proteína e água foram reportados por Schmidt \& Salas-Mellado (2009),

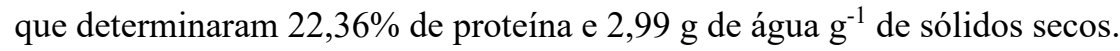

Na Figura 2, estão apresentados os valores de ganho de água (GA) das amostras ao longo do tratamento osmótico.

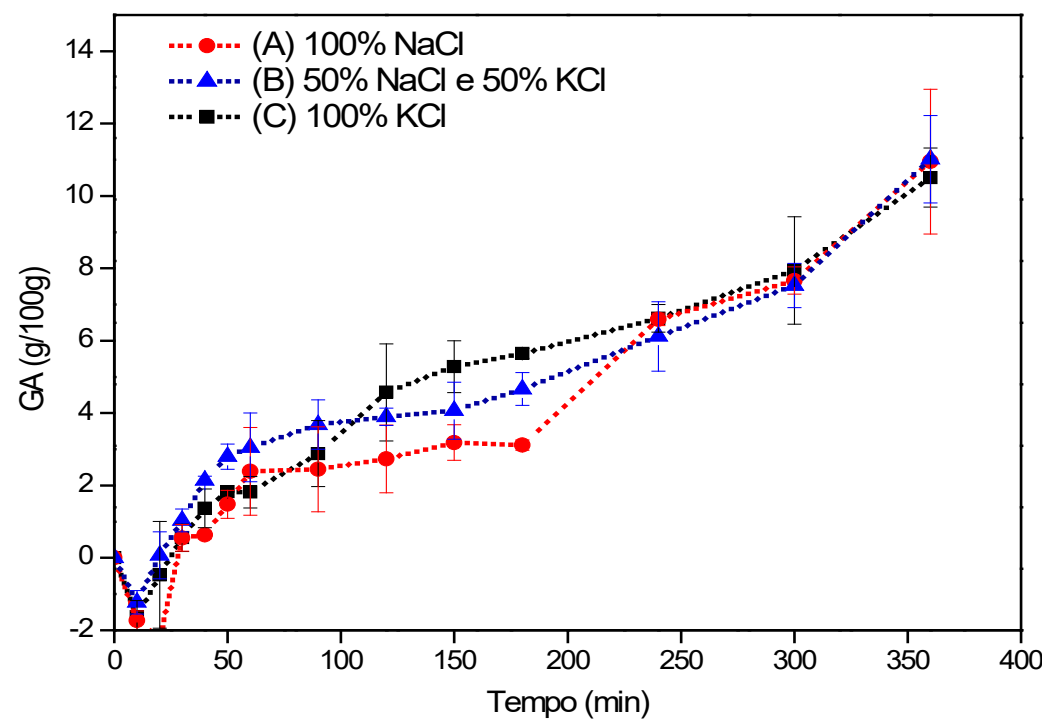

Figura 2. Valores de ganho de água utilizando salmoura com diferentes agentes osmóticos no processo de salga de cortes de peito de frango a $1{ }^{\circ} \mathrm{C}$.

Observa-se, pela Figura 2, em todos os tratamentos, um valor de $\mathrm{GA}<0$ para os primeiros 20 minutos de imersão e, a partir desse tempo, as amostras apresentaram $\mathrm{GA}>0$. O ganho de água pode estar relacionado com a penetração de soluto, o que acarretaria num aumento da pressão osmótica no interior da carne e, consequentemente, um fluxo da água no sentido oposto.

No período entre 90 e 180 minutos de processo, houve pouca variação no parâmetro GA para a formulação $(\mathrm{A})$, com diferença significativa $(p<0,05)$. Este fato pode ter ocorrido devido à menor força de repulsão eletrostática entre os filamentos de actina e miosina, quando se utiliza apenas $\mathrm{NaCl}$. Após esse período, as amostras dos diferentes tratamentos apresentaram aumento crescente no parâmetro GA, 
o que pode ser decorrência da plasmólise celular do tecido. Ao final do tratamento osmótico, para as diferentes soluções osmóticas, as amostras apresentaram incremento no ganho de água entre $10,51 \% \mathrm{e}$ $11,02 \%$, não apresentando diferenças significativas $(p>0,05)$ entre os tratamentos.

De acordo com Pardi et al. (2004), o ganho ou a perda de água de carnes em soluções salinas depende da concentração de sal e da temperatura. Assim, a baixa temperatura utilizada no experimento também pode explicar o ganho de água no processo.

Schmidt et al. (2009) verificaram que amostras de cubos de frangos, submetidas ao tratamento osmótico, absorveram água em solução com $10 \%$ de $\mathrm{NaCl}$, enquanto para concentrações entre $15 \%$ e $20 \%$, houve perda de água pelas amostras. O mesmo efeito foi observado no presente estudo para a solução com $10 \%$ de concentração de $\mathrm{NaCl}$.

A retenção de água na carne é devida à dissolução parcial da proteína e às forças de repulsão provocadas entre os íons de cloreto ligados na matriz proteica (Barat et al., 2003). Offer \& Trinick (1983) estudaram o mecanismo de retenção de água na carne e afirmaram que as miofibrilas são capazes de inchar pelo menos duas vezes o seu volume original em condições salinas que são largamente empregadas nas indústrias de carne. Estas miofibrilas ocupam cerca de $70 \%$ do volume da carne magra, o que explica a retenção de água no processamento de carne.

Nas Figuras 3 e 4, estão apresentados os dados de ganho de sal (GS) e ganho de massa (GM), respectivamente, obtidos durante o tratamento osmótico de cortes de frango em diferentes soluções salinas.

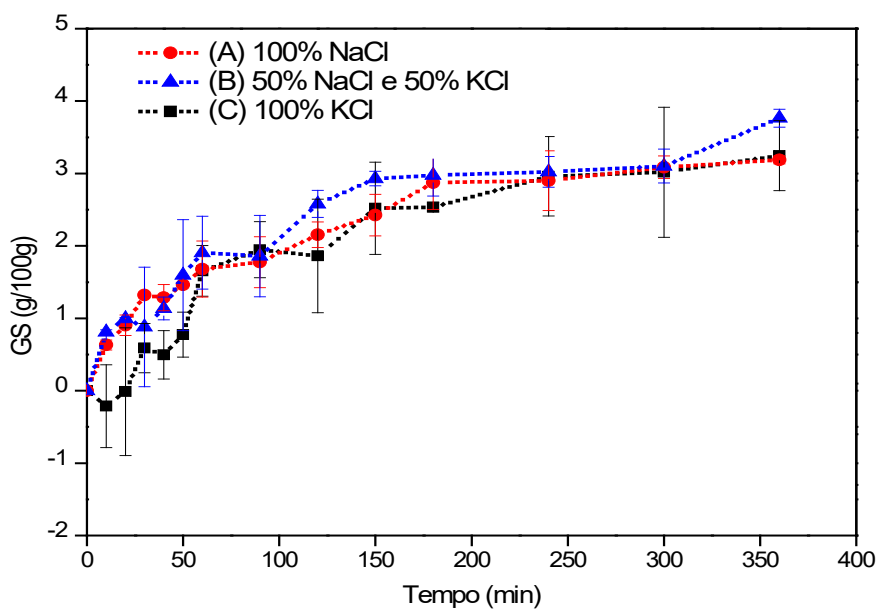

Figura 3. Valores de ganho de sal (GS) utilizando salmoura com diferentes agentes osmóticos no processo de salga de cortes de peito de frango a $1{ }^{\circ} \mathrm{C}$.

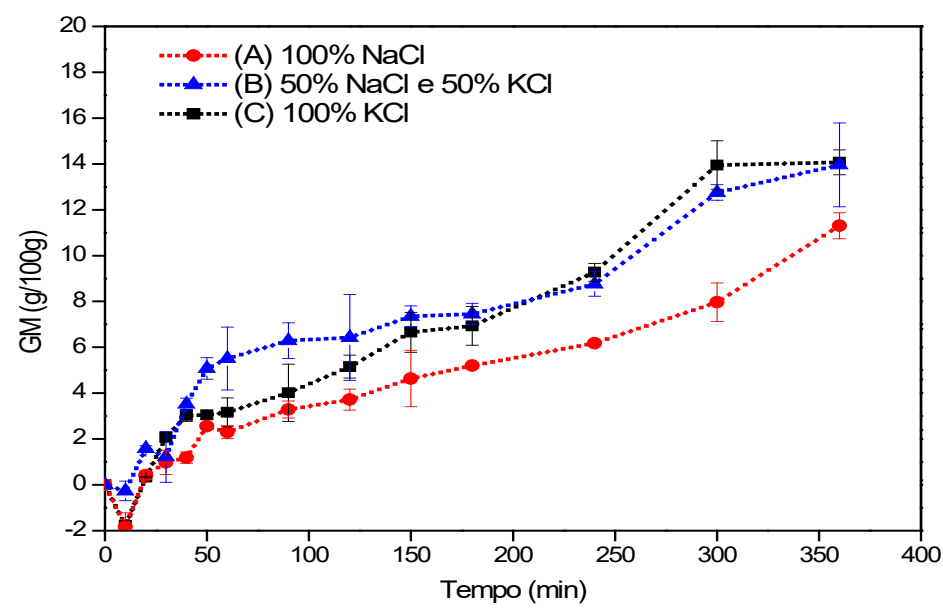

Figura 4. Valores de ganho de massa (GM) utilizando salmoura com diferentes agentes osmóticos no processo de salga de cortes de peito de frango a $1{ }^{\circ} \mathrm{C}$. 
Observa-se que, para todos os tratamentos, as amostras apresentaram aumento no parâmetro GM ao final do experimento. O tratamento com formulação (A) apresentou o GM significativamente $(p<0,05)$ menor em relação aos demais tratamentos. O aumento do parâmetro GM está relacionado com o GA e o GS das amostras, o que o explica o resultado obtido, sendo este, ao fim do tratamento, de 11,30\%, 13,96\% e 14,07\% para a formulação (A), (B) e (C), respectivamente.

As taxas de ganho de massa e ganho de sal são menores no início do processo, aumentando gradualmente. Nos primeiros minutos de processo, as amostras apresentaram valores de GS $<0$ e $\mathrm{GM}<0$. Este fato provavelmente se deve ao fato de a perda de sólidos, como proteínas solúveis, ter sido maior que a absorção de sal pelas amostras (Schmidt et al., 2008b). No caso do parâmetro GM, a perda de água das amostras no início do tratamento também teve influência sobre os valores negativos.

Com o decorrer do tempo, a penetração de sal é responsável pela formação de uma camada de soluto na superfície da carne. Essa camada se comporta como uma barreira resistente à saída de água, contribuindo para o incremento nos valores de GA e GM (Bahmani et al., 2016). O aumento nestes parâmetros também pode estar relacionado à alteração da permeabilidade da membrana celular, decorrente das mudanças físicas na estrutura da carne provocadas pelo sal. Os íons $\mathrm{Cl}^{-}$formam um complexo com as proteínas miofibrilares que proporciona um efeito de expansão dos espaços entre os filamentos de actina e miosina, aumentando a capacidade de retenção de água do músculo (Offer \& Trinick, 1983; Schmidt et al., 2008b).

Observa-se, pela Figura 3, que, na solução com a formulação (B), o valor de GS foi maior $(p>0,05)$ no final do tratamento, atingindo uma concentração de $3,77 \mathrm{~g}$ sal $100 \mathrm{~g}^{-1}$ de amostra inicial. Estudos revelam que os íons $\mathrm{K}^{+}$ penetram mais no músculo da carne do que os íons $\mathrm{Na}^{+}$(Aliño et al., 2009; Costa-Corredor et al., 2010; Barat et al., 2011). A maior entrada do íon $\mathrm{K}^{+}$pode ser decorrente da menor densidade da carga, quando comparado com o íon $\mathrm{Na}^{+}$, o que reduz a interação eletrostática com as proteínas da carne (Benmergui et al., 1979).

Barat et al. (2011) concluíram que a velocidade cinética de salga aumenta quando é utilizada uma mistura salina com KCl. Uma possível explicação para esse aumento de velocidade é a mudança do $\mathrm{pH}$ da salmoura, que implica em uma maior capacidade de retenção das proteínas da carne, devido à aproximação ao pH do ponto isoelétrico da miosina da carne.

De acordo com Martínez-Alvarez et al. (2005), a maior concentração de íons $\mathrm{K}^{+}$dissolvidos na salmoura faz com que estes se liguem de forma intensa e rápida às proteínas musculares nas camadas mais externas dos cortes de carne, provocando contração das miofibrilas e retardando a penetração dos íons cloreto. Isto justifica o fato de a formulação (C) ter se apresentado inferior à formulação (B) em relação ao GS, ao fim do tratamento. Bampi et al. (2016) relataram que amostras de corte de carne bovina tratadas com salmoura composta por $75 \% \mathrm{NaCl}$ e $25 \% \mathrm{KCl}$ apresentaram maior GS após $6 \mathrm{~h}$ de imersão, devido à maior concentração de $\mathrm{Cl}^{-}$desses cortes, quando comparadas às amostras processadas com salmoura composta de $50 \% \mathrm{NaCl}$ e $50 \% \mathrm{KCl}$.

Os valores de GS encontrados neste trabalho apresentaram-se inferiores aos valores obtidos por Schmidt et al. (2008a) durante o tratamento osmótico de carne de frango, utilizando solução com 10\% de $\mathrm{NaCl}$ a $5{ }^{\circ} \mathrm{C}$. Os autores encontraram uma concentração de $6,5 \mathrm{~g}$ sal $100 \mathrm{~g}^{-1}$ de amostra inicial em seis horas de imersão. Este fato pode estar relacionado à temperatura empregada no processo, que, no caso do presente experimento, foi de aproximadamente $1{ }^{\circ} \mathrm{C}$, sendo responsável por um efeito negativo sobre a difusão do sal. Estudos demonstram que o aumento da temperatura causa um incremento na taxa de difusão de sal para o interior do músculo da carne e ocasiona a redução da viscosidade da solução osmótica, o que diminui a resistência externa à transferência de massa (Chiralt et al., 2001; Telis et al., 2003; Tonon et al., 2006).

Na Tabela 2, estão apresentados os $D_{a p}$ da água dos cortes de frango submetidos aos diferentes tratamentos osmóticos. 
Tabela 2. Coeficientes de difusão aparente da água em cortes de frango para os diferentes tratamentos e frações mássicas de água no equilíbrio.

\begin{tabular}{ccc}
\hline Formulações & Weq $_{\text {eg de água/ g de sólidos secos) }}$ & $\boldsymbol{D}_{a p}\left(\mathbf{1 0}^{-\mathbf{1 0}} \mathbf{~ m}^{\mathbf{2}} \mathbf{s}^{\mathbf{1}}\right)$ \\
\hline$(\mathrm{A}) 100 \% \mathrm{NaCl}$ & $3,290 \pm 0,001^{\mathrm{a}}$ & $3,960 \pm 0,716^{\mathrm{b}}$ \\
\hline$(\mathrm{B}) 50 \% \mathrm{NaCl}$ e $50 \% \mathrm{KCl}$ & $3,080 \pm 0,002^{\mathrm{a}}$ & $8,051 \pm 0,744^{\mathrm{a}}$ \\
\hline$(\mathrm{C}) 100 \% \mathrm{KCl}$ & $3,295 \pm 0,003^{\mathrm{a}}$ & $5,690 \pm 0,407^{\mathrm{ab}}$ \\
\hline
\end{tabular}

As médias das colunas seguidas da mesma letra não diferem entre si, pelo teste de Tukey a $5 \%$ de probabilidade.

O $D_{a p}$ para o tratamento com a formulação (B) foi maior, apresentando diferença significativa $(p<0,05)$ apenas para a formulação (A). De acordo com Gou et al. (2004), a presença de $\mathrm{NaCl}$ na salmoura pode facilitar a circulação de água para o exterior da carne, devido ao efeito osmótico, o que acarreta um aumento da eficácia da difusividade no músculo. A presença de íons $\mathrm{K}^{+}$na solução, em concentração moderada, e a maior penetração de sal no tratamento com a formulação (B) podem explicar o maior valor do $D_{a p}$ determinado, devido ao efeito osmótico mais intenso.

Gou et al. (2004) determinaram o coeficiente de difusão aparente da água, durante o processo de cura do presunto, utilizando $\mathrm{NaCl}$, obtendo $1,2 \times 10^{-11}$ e $1,99 \times 10^{-11} \mathrm{~m}^{2} \mathrm{~s}^{-1}$, para os músculos externo e interno, respectivamente, à temperatura de $1{ }^{\circ} \mathrm{C}$. Os autores verificaram um efeito positivo da temperatura sobre o $D_{a p}$. Apesar da baixa temperatura empregada no processo, os $D_{a p}$ determinados no presente estudo apresentaram-se superiores aos valores reportados pelos autores, para os diferentes tratamentos. Isto se deve ao maior teor de água das amostras, visto que o produto sofreu hidratação no processo osmótico. RuizCabrera et al. (2004) determinaram o coeficiente de difusão aparente da água para o músculo de carne de porco durante a secagem. Os valores encontrados variaram de $3 \times 10^{-11}$ a $10^{-9} \mathrm{~m}^{2} \mathrm{~s}^{-1}$, demonstrando que a difusividade da água diminui drasticamente com a redução do teor de água, independentemente das condições experimentais.

Ao estudar a modelagem matemática do processo de difusão de $\mathrm{NaCl}$ e $\mathrm{KCl}$ durante a salga de cogumelos pré-cozidos, Bordin et al. (2019) relataram que, no processo de difusão em salmoura agitada, o nível de perturbação do sistema diminui a resistência externa formada na matriz sólida, o que foi comprovado pelo maior do número de Biot do processo agitado (600), quando comparado ao processo estático $(79,56)$. Além disso, foi demonstrado que o processo agitado confere maior mobilidade dos íons da superfície para o interior do sólido.

O fenômeno de difusividade é importante no processo de cura, uma vez que exerce influência no teor de água e na penetração de sal na carne.

\section{Conclusão}

Através dos testes realizados das diferentes formulações de salmoura, verificou-se que a formulação com $50 \% \mathrm{NaCl}$ e $50 \% \mathrm{KCl}$ apresentou maior ganho de sal pelas amostras de cortes de peito de frango, ao fim do

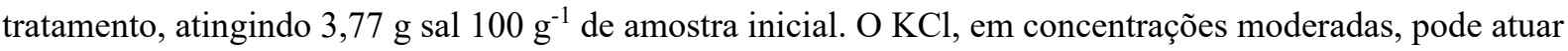
como um coadjuvante na absorção de sal e, além disso, sua utilização como substituto acarreta a redução de sódio. A temperatura e a concentração do tratamento osmótico podem estar relacionadas com o ganho de água das amostras, o qual variou entre $10,51 \%$ e $11,02 \%$, ao fim dos diferentes tratamentos. O coeficiente de difusão dos diferentes tratamentos variou de $3,960 \times 10^{-10}$ a $8,051 \times 10^{-10} \mathrm{~m}^{2} \mathrm{~s}^{-1}$, sendo o tratamento com apenas $\mathrm{NaCl}$ inferior aos demais, devido à absorção de sal pela amostra e ao efeito osmótico inferiores. A baixa temperatura empregada no processo osmótico pode ter resultado em um efeito negativo no ganho de sal.

\section{Referências}

Ahmed, I., Qazi, I. M., \& Jamal, S. (2016). Developments in osmotic dehydration technique for the preservation of fruits and vegetables. Innovative Food Science \& Emerging Technologies, 34(1), 29-43. http://dx.doi.org/10.1016/j.ifset.2016.01.003 
Aliño, M., Grau, R., Fuentes, A., \& Barat, J. M. (2010). Influence of low-sodium mixtures of salts on the post-salting stage of drycured ham process. Journal of Food Engineering, 99(2), 198-205. http://dx.doi.org/10.1016/j.jfoodeng.2010.02.020

Aliño, M., Grau, R., Toldrá, F., Blesa, E., Pagán, M. J., \& Barat, J. M. (2009). Influence of sodium replacement on physicochemical properties of dry-cured loin. Meat Science, 83(3), 423-430. PMid:20416693.

http://dx.doi.org/10.1016/j.meatsci.2009.06.022

Associação Brasileira de Proteína Animal - ABPA. (2018). Relatório anual 2018. São Paulo: ABPA.

Bahmani, A., Jafari, S. M., Shahidi, S., \& Dehnad, D. (2016). Mass transfer kinetics of eggplant during osmotic dehydration by neural networks. Journal of Food Processing and Preservation, 40(5), 815-827. http://dx.doi.org/10.1111/jfpp.12435

Bampi, M., Domschke, N. N., Schmidt, F. C., \& Laurindo, J. B. (2016). Influence of vacuum application, acid addition and partia replacement of $\mathrm{NaCl}$ by $\mathrm{KCl}$ on the mass transfer during salting of beef cuts. Lebensmittel-Wissenschaft + Technologie, 74, 2633. http://dx.doi.org/10.1016/j.Iwt.2016.07.009

Barat, J. M., Baigts, D., Aliño, M., Fernández, F. J., \& Pérez-García, V. M. (2011). Kinetics studies during NaCl and $\mathrm{KCl}$ pork meat brining. Journal of Food Engineering, 106(1), 102-110. http://dx.doi.org/10.1016/j.jfoodeng.2011.04.022

Barat, J. M., Rodriguez-Barona, S., Andrés, A., \& Fito, P. (2003). Cod salting manufacturing analysis. Food Research International, 36(5), 447-453. http://dx.doi.org/10.1016/S0963-9969(02)00178-3

Benmergui, E. A., Fontan, C. F., \& Chirife, J. (1979). The prediction of water activity in aqueous solutions in connection with intermediate moisture foods I. aw Prediction in single aqueous electrolyte solutions. International Journal of Food Science \& Technology, 14(1), 625-637. http://dx.doi.org/10.1111/j.1365-2621.1979.tb00909.x

Bordin, M. S. P., Borsato, D., Cremasco, H., Galvan, D., Silva, L. R. C., Romagnoli, É. S., \& Angilelli, K. G. (2019). Mathematical modeling of multicomponent $\mathrm{NaCl}$ and $\mathrm{KCl}$ diffusion process during the salting of pre-cooked champignon mushrooms. Food Chemistry, 273, 99-105. PMid:30292382. http://dx.doi.org/10.1016/j.foodchem.2018.01.188

Chen, J., Hu, Y., Wen, R., Liu, Q., Chen, Q., \& Kong, B. (2019). Effect of $\mathrm{NaCl}$ substitutes on the physical, microbial and sensory characteristics of Harbin dry sausage. Meat Science, 156, 205-213. PMid:31202095. http://dx.doi.org/10.1016/j.meatsci.2019.05.035

Chiralt, A., Fito, P., Barat, J. M., Andrés, A., González-Martínez, C., Escriche, I., \& Camacho, M. M. (2001). Use of vacuum impregnation in food salting process. Journal of Food Engineering, 49(2-3), 141-151. http://dx.doi.org/10.1016/S02608774(00)00219-3

Costa-Corredor, C., Muñoz, I., Arnau, J., \& Gou, P. (2010). Ion uptakes and diffusivities in pork meat brine-salted with $\mathrm{NaCl}$ and K-lactate. Food Science and Technology, 43(8), 1226-1233.

Crank, J. (1975). The mathematics of diffusion (2nd ed.). Oxford: Clarendon Press.

Desmond, E. (2006). Reducing salt: A challenge for the meat industry. Meat Science, 74(1), 188-196. PMid:22062728. http://dx.doi.org/10.1016/j.meatsci.2006.04.014

Gou, P., Comaposada, J., \& Arnau, J. (2004). Moisture diffusivity in the lean tissue of dry-cured ham at different process times. Meat Science, 67(2), 203-209. PMid:22061315. http://dx.doi.org/10.1016/j.meatsci.2003.10.007

Instituto Adolfo Lutz - IAL. (2008). Métodos físico-químicos para análise de alimentos (4. ed.). São Paulo: IAL.

Kloss, L., Meyer, J. D., Graeve, L., \& Vetter, W. (2015). Sodium intake and its reduction by food reformulation in the European Union: A review. Official Journal of the Society of Nutrition and Food Science, 1, 9-19.

http://dx.doi.org/10.1016/j.nfs.2015.03.001

Lazic, I. B., Raseta, M., Nikolic, D., Lukic, M., Karan, G., \& Lilic, S. (2015). Reducing the sodium chloride content in chicken pate by using potassium and ammonium chlorie. Procedia Food Science, 5, 22-25. http://dx.doi.org/10.1016/j.profoo.2015.09.006

Lilic, S., Matekalo-Sverak, V., \& Borovic, B. (2008). Possibility of possibility of replacement of sodium chloride by potassium chloride in cooked sausages: Sensory characteristics and health aspects. Biotechnology in Animal Husbandry, 24(1-2), 133138. http://dx.doi.org/10.2298/BAH0802133L

Martínez-Alvarez, O., Borderías, A. J., \& Gómez-Guillén, M. C. (2005). Sodium replacement in the cod (Gadus morhua) muscle salting process. Food Chemistry, 93(1), 125-133. http://dx.doi.org/10.1016/j.foodchem.2004.10.014

Nascimento, R., Campagnol, P. C. B., Monteiro, E. S., \& Pollonio, M. A. R. (2007). Substituição de cloreto de sódio por cloreto de potássio: Influência sobre as características físico-químicas e sensoriais de salsichas. Alimentos e Nutrição, 18(3), 297-302.

Offer, G., \& Trinick, J. (1983). On the mechanism of water holding in meat: The swelling and shrinking of myofibrils. Meat Science, 8(4), 245-281. PMid:22055626. http://dx.doi.org/10.1016/0309-1740(83)90013-X

Pardi, M. C., Santos, I. F., \& de Souza, E. R. (2004). Ciência, higiene e tecnologia de carnes (2. ed.). Goiânia: UFG.

Paulino, F. O., Silva, T. J. P., Franco, R. M., Freitas, M. Q., \& Fernandes, M. L. (2006). Redução parcial dos teores de gordura e sal em embutido cárneo suíno com utilização de goma carragena e cloreto de potássio. Revista Brasileira de Ciência Veterinária, 13(2), 121-124. http://dx.doi.org/10.4322/rbcv.2014.283

Rastogi, N. K., \& Raghavarao, K. S. M. S. (2004). Mass transfer during osmotic dehydration determination of moisture and solute diffusion coefficients from concentration profiles. Food and Bioproducts Processing, 82(1), 44-48. http://dx.doi.org/10.1205/096030804322985308

Rocha Garcia, C., Bolognesi, V. J., \& Shimokomaki, M. (2013). Aplicações tecnológicas e alternativas para redução do cloreto de sódio em produtos cárneos. Boletim do Centro de Pesquisa e Processamento de Alimentos, 31(1), 139-150.

Ruiz-Cabrera, M. A., Gou, P., Foucat, L., Renou, J. P., \& Daudin, J. D. (2004). Water transfer analysis in pork meat supported by NMR imaging. Meat Science, 67(1), 169-178. PMid:22061130. http://dx.doi.org/10.1016/j.meatsci.2003.10.005 
Estudo da transferência de massa de cortes de frango durante o tratamento osmótico em diferentes soluções salinas Stevanato, N., \& Porciuncula, B. D. A.

Ruusunen, M., \& Puolanne, E. (2005). Reducing sodium intake from meat products. Meat Science, 70(1), 531-541. PMid:22063751. http://dx.doi.org/10.1016/j.meatsci.2004.07.016

Sabadini, E., Carvalho Junior, B. C., Sobral, P. J. A., \& Hubinger, M. D. (1998). Mass transfer and diffusion coefficient determination in wet and dry salting of meat. Drying Technology, 16(9-10), 2095-2115. http://dx.doi.org/10.1080/07373939808917514

Sabadini, E., Hubinger, M. D., Sobral, P. J. A., \& Carvalho Junior, B. C. (2001). Alterações da atividade de água e da cor da carne no processo de elaboração da carne salgada desidratada. Food Science and Technology, 21(1), 14-19.

Schmidt, C. G., \& Salas-Mellado, M. (2009). Influência da ação das enzimas alcalase e flavourzyme no grau de hidrólise das proteínas de carne de frango. Quimica Nova, 32(5), 1144-1150. http://dx.doi.org/10.1590/S0100-40422009000500012

Schmidt, F. C., Carciofi, B. A. M., \& Laurindo, J. B. (2008a). Efeito da impregnação a vácuo na transferência de massa durante o processo de salga de cortes de peito de frango. Food Science and Technology, 28(2), 366-372. http://dx.doi.org/10.1590/S0101-20612008000200015

Schmidt, F. C., Carciofi, B. A. M., \& Laurindo, J. B. (2008b). Salting operational diagrams for chicken breast cuts: Hydrationdehydration. Journal of Food Engineering, 88(1), 36-44. http://dx.doi.org/10.1016/j.jfoodeng.2007.12.005

Schmidt, F. C., Carciofi, B. A. M., \& Laurindo, J. B. (2009). Application of diffusive and empirical models to hydration, dehydration and salt gain during osmotic treatment of chicken breast cuts. Journal of Food Engineering, 91(4), 553-559. http://dx.doi.org/10.1016/j.jfoodeng.2008.10.003

Silva Sobrinho, A. G., Zeola, N. M. B. L., Souza, H. B. A., \& Lima, T. M. A. (2004). Qualidade da carne ovina submetida ao processo de salga. Food Science and Technology, 24(3), 369-372. http://dx.doi.org/10.1590/S0101-20612004000300011

Stanley, R. E., Bower, C. G., \& Sullivan, G. A. (2017). Influence of sodium chloride reduction and replacement with potassium chloride based salts on the sensory and physico-chemical characteristics of pork sausage patties. Meat Science, 133, 36-42. PMid:28599172. http://dx.doi.org/10.1016/j.meatsci.2017.05.021

Telis, V. R. N., Romanelli, P. F., Gabas, A. L., \& Telis-Romero, J. (2003). Salting kinetics and diffusities in farmed caiman muscle. Pesquisa Agropecuária Brasileira, 28(4), 529-535. http://dx.doi.org/10.1590/S0100-204X2003000400012

Tonon, R. V., Baroni, A. F., \& Hubinger, M. D. (2006). Estudo da desidratação osmótica de tomate em soluções ternárias pela metodologia de superfície de resposta. Food Science and Technology, 26(3), 715-723. http://dx.doi.org/10.1590/S010120612006000300036

Verma, A. K., \& Banerjee, R. (2012). Low-sodium meat products: Retaining salty taste for sweet health. Critical Reviews in Food Science and Nutrition, 52(1), 72-84. PMid:21991991. http://dx.doi.org/10.1080/10408398.2010.498064

Vidal, V. A. S., Biachi, J. P., Paglarini, C. S., Pinton, M. B., Campagnol, P. C. B., Esmerino, E. A., da Cruz, A. G., Morgano, M. A., \& Pollonio, M. A. R. (2019). Reducing 50\% sodium chloride in healthier jerked beef: An efficient design to ensure suitable stability, technological and sensory properties. Meat Science, 152, 49-57. PMid:30802818. http://dx.doi.org/10.1016/j.meatsci.2019.02.005

Zheng, J., Han, Y., Ge, G., Zhao, M., \& Sun, W. (2019). Partial substitution of NaCl with chloride salt mixtures: Impact on oxidative characteristics of meat myofibrillar protein and their rheological properties. Food Hydrocolloids, 96, 36-42. http://dx.doi.org/10.1016/j.foodhyd.2019.05.003

Ziebert, R. A., \& Skikida, P. F. A. (2004). Avicultura e produção integrada em Santa Helena, estado do Paraná: Uma abordagem a partir da nova economia institucional. Agricultura em São Paulo, 15(1), 71-86. 